\title{
Quaderni
}

QUADERNI Communication, technologies, pouvoir

79 | Automne 2012

Produire la démocratie

\section{Des assemblages incertains aux technologies de démocratie : deux modalités de l'expérience démocratique}

\section{Brice Laurent}

\section{(2) OpenEdition \\ Journals}

Édition électronique

URL : http://journals.openedition.org/quaderni/623

DOI : 10.4000/quaderni.623

ISSN : 2105-2956

Éditeur

Les éditions de la Maison des sciences de l'Homme

\section{Édition imprimée}

Date de publication : 5 octobre 2012

Pagination : 81-98

Référence électronique

Brice Laurent, « Des assemblages incertains aux technologies de démocratie : deux modalités de l'expérience démocratique ", Quaderni [En ligne], 79 | Automne 2012, mis en ligne le 05 octobre 2014, consulté le 05 mai 2019. URL : http://journals.openedition.org/quaderni/623 ; DOI : 10.4000/ quaderni.623 


\section{$D$ ossier}

Des assemblages incertains aux technologies de démocratie : deux modalités de l'expérience démocratique

\section{Brice Laurent}

Chargé de recherche Centre de Sociologie de l'Innovation Mines Paris Tech
La pratique démocratique est réalisée par des canaux divers. Le suffrage universel en fait partie, mais aussi, à des titres différents, de nombreux dispositifs dits «participatifs", plus ou moins formalisés dans le droit, et faisant aujourd'hui l'objet d'un vaste travail analytique. Pour les chercheurs intéressés par le renouvellement des pratiques démocratiques comme les acteurs impliqués dans des tentatives à la légitimité incertaine, les initiatives participatives peuvent souvent être décrites comme des « expériences ». Un récent ouvrage décrit ainsi la procédure dont la Commission Nationale du Débat Public a la charge comme une « expérience française de démocratie participative ${ }^{1}$. Des élus locaux devenus commanditaires de conférences de citoyens rendent publique "l'expérience démocratique » qu'ils ont contribué à réaliser ${ }^{2}$. La référence à l'expérience indique une stabilisation non acquise du dispositif, une certaine incertitude sur ses résultats et ses possibilités de traduction dans la fabrique de la décision collective. Elle indique aussi que peut-être l'initiative donnera lieu à de futurs développements.

Cet article propose de prendre au sérieux la qualification d' « expérience » pour les pratiques démocratiques, afin d'en analyser les contours et les manifestations politiques. Il développe la notion d' « expérience » pour proposer une analyse des initiatives participatives explicitant les constructions démocratiques auxquelles elles donnent lieu. Il contraste pour ce faire deux figures de la pratique démocratique susceptibles d'être décrites par le vocabulaire de l'expérience. L'une prend la forme d'un " assemblage » caractérisé par l'exploration d'une situation d'indétermination. L'autre est fondée sur la 
reproduction d'un instrument qui peut être qualifié de « technologie de démocratie ». Dans les deux cas, le parallèle avec l'expérience scientifique est instructif. En s'appuyant sur un ensemble de travaux de sociologie des sciences, l'analyse des deux modalités de l'expérience démocratique permettra d'élaborer un répertoire de description rendant compte des situations pratiques de la participation et des ordres démocratiques qu'elles produisent.

L'objectif ici est non seulement de suggérer des outils de description et d'analyse de la « participation du public » et des experts qui la font vivre, mais aussi de contribuer à une étude de la démocratie attentive aux dispositifs par l'intermédiaire desquels se constitue la légitimité des opérations politiques, et les modes d'action des publics de la démocratie libérale ${ }^{3}$. Par « dispositif», on entend ici l'ensemble des instruments, standardisés ou non, qui mettent en forme des modes d'action pour le citoyen en même temps qu'ils définissent des problèmes légitimes. Par exemple, l'étude des dispositifs matériels permettant de réaliser l'opération électorale et de la publicisation de certaines parties du mécanisme montre comment l'organisation pratique du vote met en forme un système politique définissant des pratiques acceptables. Ces processus sont explicités par les travaux analysant la mise en place des mécanismes du suffrage universel ${ }^{4}$, mais aussi par les études des controverses plus récentes qui permettent de mettre au jour les logiques nationales de la production de l'ordre démocratique à travers les dispositifs électoraux ${ }^{5}$.

Ces travaux incitent à rendre compte des dispositifs «expérimentaux » réalisant les pratiques démocratiques, ce que cet article choisit de faire en discutant la notion même d' " expérience $»^{6}$. Pour ce faire, il s'appuie sur des cas relatifs aux nanotechnologies ${ }^{7}$. Le domaine est intéressant pour plusieurs raisons. Première raison, les nanotechnologies sont un domaine construit à la suite de grands programmes de politique scientifique, et marqué par des incertitudes fortes sur la caractérisation des objets techniques et de leur risque, mais aussi sur l'identité des publics concernés. À ce titre, les nanotechnologies constituent une épreuve originale pour la pratique démocratique, qui permet, du fait de ces spécificités, de rendre visibles les investissements nécessaires à l'organisation des expériences démocratiques. Les exemples analysés ici sont donc très particuliers, mais l'hypothèse sur laquelle cet article se fonde est que ces particularités mêmes, en imposant une interrogation explicite sur les expériences démocratiques, permettent d'en étudier les modalités. Ceci est d'autant plus pertinent que les programmes de soutien au développement des nanotechnologies - et c'est une seconde raison de s'y intéresser ici - ont fait la part belle, dès leurs premières formulations, au thème de la «participation» et de « l'engagement » du « public ». S'en sont suivies de multiples initiatives participatives. En France, le conseil régional de l'Île-de-France a fait organiser par l'IFOP une conférence de citoyens en $2007^{8}$. Un débat public national a été organisé en 2009 par la Commission Nationale du Débat Public. Commandité par le gouvernement, il avait pour but « d'éclairer la décision publique »sur les choix politiques liés aux nanotechnologies. À cela s'ajoutent des dispositifs hybrides et originaux. C'est le 
cas du Nanoforum, organisé au Conservatoire National des Arts et Métiers avec le soutien de la Direction Générale de la Santé (DGS), qui rassemble entre 2007 et 2009 des associations et des acteurs administratifs, dans le but de rendre explicites les incertitudes sur les choix publics relatifs à la définition des programmes ou à la réglementation des nanotechnologies. C'est aussi celui d'une initiative de l'Association Française de Normalisation (AFNOR), qui consiste à construire un dispositif (une « norme nano-responsable ») permettant aux producteurs de nanomatériaux de faire circuler les informations sur leurs produits, y compris lorsqu'elles ont trait à l'incertitude sur leurs risques potentiels, et à prendre en compte les attentes des publics intéressés à mesure qu'ils se manifestent. Les modalités de la « participation » sont souvent controversées. Les discussions autour des nanotechnologies en France ont donné lieu à l'organisation de nombreux « contre-débats », notamment lors du débat public CNDP, dont la plupart des réunions publiques ont été interrompues par des opposants au développement des nanotechnologies, qui voyaient dans ce débat une manœuvre pour faire accepter des décisions déjà prises ${ }^{9}$.

Ces exemples sont autant d' « expériences démocratiques » par l'intermédiaire lesquelles les rôles de publics variés et les modalités de la participation légitime sont définis. Caractériser la nature de ces expériences permettra de disposer d'un répertoire d'analyse pour observer la démocratie en train de se faire, plutôt que de juger des dispositifs sur la base de « critères démocratiques » connus à l'avance. Pour ce faire, cet article propose de considérer dans une première section l'expérience comprise comme assemblage incertain, puis, dans une seconde, l'expérience comprise comme utilisation d'une " technologie de démocratie » susceptible de circuler. Le passage de l'une à l'autre forme d'expérience implique le passage d' « épreuves de réplication », qui seront abordées dans la troisième et dernière section.

Dans la mesure où l'objectif est ici de rendre compte de la variété des formes démocratiques et non pas de définir des critères pour l'évaluation des procédures ou des pratiques collectives, il est possible de se contenter d'une identification large des pratiques démocratiques, qui ne cherche pas à séparer en amont de l'analyse les procédures participatives «vraiment » démocratiques d'autres qui ne le seraient pas. On peut considérer à ce stade que les pratiques démocratiques ont trait à l'organisation publique des oppositions entre individus ou groupes, tout en maintenant un degré d'indétermination sur les évolutions de la vie collective ${ }^{10}$. Néanmoins, nous verrons que la question critique et normative (qu'est ce qui est démocratique et qu'est-ce qui ne l'est pas ?), peut être reposée à l'issue de cette analyse - non pas en imposant un critère d'évaluation de procédures définies ex ante comme " participatives », mais en repensant ce que peut être la critique de la démocratie.

\section{L'expérience comme assemblage incertain}

Une première modalité de l'expérience démocratique est illustrée par des initiatives dont les organisateurs font face à une incertitude relative aux objectifs mêmes des dispositifs mis en place. Le Nanoforum, par exemple, est organisé par un groupe de personnes réunies 
dans un comité de pilotage chargé de préparer les réunions publiques, de rédiger des « fiches de problématiques », et d'évaluer les discussions. Ce comité comprend des représentants des différents ministères concernés, d'une association de protection de l'environnement (France Nature Environnement), d' « experts qualifiés » (en sciences physiques et en sciences sociales) et de l'association Vivagora, qui milite pour la « mise en démocratie » de l'innovation, et qui est à l'origine de l'initiative avec William Dab, ancien directeur général de la santé et professeur au CNAM. Le comité cherche à inventer de « nouvelles formes de discussion »sur l'évaluation des risques et des bénéfices des nanotechnologies (Dab et al., 2009). Le Nanoforum n'est pas pensé comme une instance vouée à produire des recommandations à l'usage des pouvoirs publics, mais comme un lieu d'explicitation des problèmes à traiter par les acteurs administratifs. Cet objectif est mis en œuvre par le biais de réunions publiques, organisées à Paris, qui abordent une variété de thèmes. Certaines sont consacrées à une série de secteurs industriels, dont des représentants sont invités à présenter leur utilisation des nanomatériaux. D'autres prennent pour objet l'organisation de la recherche locale, à Grenoble notamment ${ }^{11}$. Enfin, un cycle de réunions est consacré aux particules d'argent à l'échelle nanométrique (le nano-argent).

Le fait de centrer les discussions sur le nanoargent permet d'examiner dans le détail les problèmes de l'évaluation risques-bénéfices ou de l'étiquetage dans une situation où la définition des objets même (ce qui fait le caractère « nano » du nano-argent) n'est pas certaine. En effet, les propriétés liées à la taille (et notamment les dangers potentiels) peuvent certes dépendre du diamètre des particules, mais aussi de leur surface spécifique, c'est-à-dire leur surface susceptible de donner lieu à des réactions chimiques. Dans le cas du nano-argent, les conclusions du Nanoforum ne recommandent aucune décision tranchée, mais elles contribuent, pour les participants aux réunions publiques et au comité de pilotage, à identifier les problèmes que les pouvoirs publics ont à traiter. Ainsi, la plupart des membres du comité de pilotage, et notamment les représentants de la Direction Générale de la Santé, sont à l'origine de l'initiative menée à l'Association Française de Normalisation (AFNOR) visant à développer une norme dite « nano-responsable », qui fournirait aux industriels producteurs de substances « nano » la possibilité de faire circuler des informations sur les objets qu'ils produisent ou distribuent. Dans la situation d'incertitude sur la caractérisation des objets, la norme nano-responsable se veut un outil de prise de décision en situation d'incertitude, en même temps qu'un dispositif flexible permettant d'adapter les informations fournies à l'avancée des recherches. Elle est pensée comme un outil destiné à l'ensemble des « parties prenantes concernées » (fournisseurs, clients, distributeurs, riverains, consommateurs...). Le projet est mené, en conséquence, par un ensemble d'acteurs industriels, administratifs et associatifs et est pensé par ses initiateurs comme une « expérience permettant de rassembler l'ensemble des attentes pour la production responsable des nanomatériaux $»^{12}$.

À l'heure actuelle, le Nanoforum est un dispositif qui n'a pas été pérennisé. Sur la base des versions préliminaires de la norme « nano-responsable », 
la France est parvenue à faire accepter ce projet au Comité Européen de Normalisation (CEN) depuis 2011, où il entre dans la démarche d' ' innovation responsable » dans les nanotechnologies ${ }^{13}$. Les deux initiatives sont liées par les intervenants impliqués dans l'une et l'autre. Elles ont d'autres points communs, et notamment le type d' « expérience participative » qu'elles proposent. Dans ces deux cas, l'expérience est à comprendre au sens des pragmatistes américains, pour qui elle caractérise la situation dans laquelle le monde lui-même s'invente et s'éprouve. Les conclusions auxquelles l'expérience parvient peuvent alors être plus ou moins stables, et en tout cas toujours "sujettes à être modifiées au cours de l'expérience future” (James, 2005 : 33). Dans cette perspective pragmatiste, l'expérience désigne le processus graduel qui ne sépare pas la réflexion et l'action, l'engagement du sujet et de l'objet mais les contient « dans une même totalité » pour finalement amener une nouvelle réalité à l'existence (Dewey, 1958: 8). Elle pointe vers des lieux où les objectifs et les voies pour les atteindre ne sont pas donnés, mais sont à construire par les acteurs impliqués. Les organisateurs et les participants du Nanoforum savent, au départ, qu'ils veulent rendre explicites les problèmes suscités par les nanotechnologies que les pouvoirs publics devront traiter. Les initiateurs du projet « nano-responsable » définissent l'ambition initiale par la nécessité d'identifier collectivement et (suivant l'expression de l'initiatrice du projet à la DGS) « de façon pluraliste », des approches pour « traiter l'incertitude sur les risques et les bénéfices des nanomatériaux ». Mais la formulation même de ces objectifs contient la nécessité de l'expérience comme exploration d'un monde non donné a priori, du fait même de la définition non stabilisée des programmes et des objets des nanotechnologies.

Le cas des nanotechnologies est un cas très particulier, marqué par une très forte indétermination. Il permet d'identifier une modalité de l'expérience démocratique au sein de laquelle l'expérience est ici un assemblage original, dont la stabilité n'est pas acquise, entre des individus, des objets à définir et des programmes en constitution. Cet assemblage ne repose pas sur une séparation a priori entre un instrument « participatif » (au sens où il organiserait la «participation » d'un ou des publics) et son application à une question donnée à l'avance, mais une expérience collective, au sein de laquelle les rôles des uns et des autres s'inventent, et font face à une multitude de contraintes qu'ils découvrent.

La nature de ces assemblages implique en effet que de multiples contraintes émergent au cours de l'expérience. Ces contraintes peuvent être liées aux particularités des objets discutés. Par exemple, le nano-argent se révèle une entité insaisissable, transformée lors de sa circulation dans les milieux naturels, et dont les connexions entre les caractéristiques physico-chimiques et les risques potentiels ne sont pas tracées. Les contraintes ont également trait à la confrontation des intérêts des acteurs impliqués. Par exemple, au cours des discussions à l'AFNOR, les réticences de certains participants industriels à la certification (et donc à la possibilité d'afficher publiquement le respect de la norme nanoresponsable) sont le signe du refus de la part de certains industriels de s'engager trop loin dans la voie de la « responsabilité ». 
L'expérience fondée sur les technologies de démocratie

Comprendre l'expérience comme assemblage et invention du monde incite à faire le lien avec la science expérimentale, et notamment avec l'expérience scientifique comme situation fondée sur la mobilisation de dispositifs techniques permettant de «parler pour la nature " via des démonstrations publiques. L'histoire et la sociologie des sciences ont fourni des études détaillées de la pratique scientifique qui suggère de compléter la description de l'expérience comme assemblage incertain par une autre modalité de celle-ci. Un exemple phare de la pratique de la science expérimentale est celui de Robert Boyle, l'inventeur de la pompe à air ${ }^{14}$. Lorsque Boyle met au point la pompe à air, il redéfinit par là le problème de l'existence du vide, qui agite alors les cercles intellectuels de l'époque : la question n'est plus de disserter sur la nature ontologique du vide, mais de constater quels effets sont produits par un dispositif technique qui élimine l'air d'un volume défini. En inventant la pompe à air, Boyle « expérimente » avec le réel, qu'il contribue à produire. C'est une situation comparable aux assemblages décrits plus haut, dans laquelle le rôle du " savant ", en train d'inventer les principes de la science physique moderne, la nature des objets qu'il manipule ${ }^{15}$ et les connaissances qu'il produit ne sont pas donnés, mais s'expérimentent.

Mais Boyle fait aussi de la pompe à air une technologie qui circule, qui doit permettre de reproduire des opérations qu'il décrit dans ses ouvrages, des « expériences types » dont la reproductibilité est le gage de la valeur de l'instrument. Boyle associe donc étroitement l'expérience comme invention d'un monde en cours de définition à l'expérience comme situation paramétrée dont on jugera la valeur suivant la correspondance avec un résultat conforme aux expériences précédentes. Ce faisant, il utilise la pompe à air afin de «parler pour la nature » par l'intermédiaire d'une technologie scientifique qui associe une instrumentation matérielle (la pompe à air elle-même), une organisation sociale (qui définit qui sont les témoins acceptables susceptibles d'attester de la validité de l'expérience) et une manière d'écrire les comptes-rendus. La valeur de l'expérience est donc étroitement liée à la possibilité de la démonstration publique à laquelle elle donne lieu.

On peut donc contraster les cas d'expériences démocratiques comme assemblages incertains avec d'autres, qui sont aussi des « expériences », mais réalisées sur le modèle de l'expérience scientifique fondée sur l'utilisation d'un instrument stabilisé, permettant de faire parler le public, de même que les instruments scientifiques parlent pour la nature ${ }^{16}$. Par exemple, la conférence de citoyens (et en particulier celle qui nous intéressera ici) est censée constituer un public fait d'individus « neutres », non connaisseurs des questions traitées et non intéressés par lui, afin d'assurer ses délibérations sur un sujet présenté lors des séances de formation de la façon « la plus objective possible ». Le dispositif est ici censé permettre de porter la voix d'une double objectivité : celle du " citoyen neutre », et celle de l'information scientifique qui lui est présentée. Dans ce cas, l'expérience consiste à tenter de faire produire, par un panel de citoyens neutres, des recommandations « raisonnables », 
« acceptables » par les parties prenantes du débat public. Un autre exemple de cette modalité de l'expérience démocratique est le débat public de la CNDP, qui cherche à faire intervenir les publics intéressés, de façon la plus large possible, tout en permettant aux « parties prenantes » qui le souhaitent de distribuer un " cahier d'acteur », dans lequel elles présentent leur position sur le sujet traité. La représentation des arguments et des publics constitués est l'objectif de l'instrument et l'expérience désigne la tentative de production d'une telle cartographie. À l'instrument est associée dans ces deux cas une organisation sociale permettant d'assurer la valeur de l'expérience. Les instruments participatifs que sont la conférence de citoyen et le débat public CNDP s'appuient sur des expertises, elles-mêmes liées aux sciences sociales. Par exemple celle de l'institut de sondage organisateur de conférences, comme l'IFOP, dans un cas, celle des membres de la Commission Nationale du Débat Public et des Commissions Particulières chargées de chaque débat, dans l'autre. Les employés de l'IFOP en charge des conférences de citoyens sont des lecteurs de la littérature consacrée aux conférences de citoyens, et prennent soin d'inclure des spécialistes de la participation dans les comités de pilotage des conférences qu'ils organisent. Les Commissions Particulières chargés de l'organisation des débats CNDP comprennent fréquemment des chercheurs. Enfin, comme l'expérience scientifique, ce type d'expérience démocratique s'appuie sur des procédés d'écriture qui permettent de produire des recommandations écrites par les citoyens du panel de la conférence de citoyens, ou les verbatim assurant la publicité du débat CNDP et la traçabilité de l'avis final de la Commission.
L'association entre l'instrument, l'organisation sociale qu'il implique et les procédés d'écriture sur lesquels il repose peut être définie comme une « technologie de démocratie $»^{17}$. De même que la technologie scientifique définit les procédures permettant de produire le fait objectif, la technologie de démocratie définit les procédures permettant de produire la pratique démocratique. À un critère de vérité répond donc un critère de démocratie, dont la manipulation est réservée à des experts : un institut de sondage qui commercialise la conférence de citoyens comme outil de production d'avis " mesurée " par des citoyens «neutres » dans le premier cas, et, dans le second, une autorité administrative indépendante ayant défini son rôle par l'organisation de dispositifs qu'elle a contribué à réaliser.

De même que pour l'expérience scientifique, la technologie de démocratie est liée à une démonstration. Les démonstrations réalisées par ces technologies sont liées à l'explicitation de la valeur des productions du dispositif et de l'acceptabilité du processus adopté pour y parvenir. Pour les commanditaires, cela revient à publiciser l'expérience pour démontrer son intérêt. La conférence de citoyens sur les nanotechnologies du conseil régional de l'îlede-France, par exemple, a donné lieu à un film (Les Nanos et nous), traitant à la fois de la procédure et du sujet discuté, dont le coût a été vivement discuté au sein du conseil régional de l'île-de-France, mais qui était une composante importante de la visibilité de l'expérience ${ }^{18}$. Pour les experts du dispositif, la démonstration est cruciale. Les intérêts marchands de l'IFOP sont explicites : comme l'institut vend la conférence de citoyens à des entreprises et à 
des collectivités locales, il doit démontrer la valeur du dispositif et sa capacité à produire ce qu'attendent les commanditaires, c'est-à-dire des recommandations acceptables produites par un public fait de citoyens «neutres ». À cet égard, l'IFOP est satisfait des démonstrations successives auprès de ses clients ${ }^{19}$. La CNDP quant à elle doit mettre en évidence le paysage des arguments échangés sur un sujet donné, et donc faire en sorte que les acteurs intéressés rédigent des cahiers d'acteurs et interviennent lors des réunions publiques. La transmission des débats sur internet et la diffusion des verbatim sont autant de façons de rendre visibles les processus de discussion collective. L'expérience est alors moins validée par l'explicitation de ses « impacts » sur la prise de décision publique que par la qualité de la démonstration. Mais les deux ne sont pas contradictoires, tout particulièrement du côté des commanditaires. Ainsi, le conseiller régional d'Île-de-France à l'origine de la commande à l'IFOP de la conférence de citoyens sur les nanotechnologies prend soin d'écrire à chacun des membres du panel quelques mois après la conférence, afin d'expliquer quelles recommandations ont été prises en compte (notamment des financements régionaux pour la recherche en toxicologie) et lesquelles n'ont pu l'être ${ }^{20}$.

L'expérience sur la technologie de démocratie et les démonstrations qu'elle implique sont censées assurer que l'instrument produit le résultat souhaité, c'est-à-dire, dans les exemples utilisés ici, un avis raisonnable produit par un citoyen neutre, et l'exposé des arguments des publics concernés. L'usage des technologies de démocratie conduit à mobiliser des experts d'un instrument, à assurer des démonstrations publiques, et à éprouver la résistance de la technologie aux critiques. En contrastant cette version de l'expérience avec la première (l'expérience comme exploration incertaine), on voit toute l'importance de s'interroger sur les conditions de possibilité de la séparation entre une technologie de démocratie et les « cas » singuliers sur lesquels elle s'applique - cela même que Boyle parvient à réaliser en faisant circuler la pompe à air, passant ainsi de l'expérience comme incertitude radicale à l'expérience comme reproduction de l'instrument scientifique stabilisé. Ceci implique de se pencher sur les tentatives effectuées par les experts des technologies de démocratie d'arracher un instrument d'un assemblage local afin de parvenir à le faire circuler.

\section{De l'assemblage à la technologie de démocratie : épreuves de réplication}

Comment assurer le passage des assemblages successifs à la solidification d'un instrument (qu'il soit une technologie scientifique ou une technologie de démocratie) capable de résister à ses multiples réplications ? La sociologie des sciences a montré que la stabilisation des instruments scientifiques passe par la solidification de standards et de références permettant la transformation de l'assemblage ponctuel en «boite-noire », qui peut faire de la connaissance scientifique un « mobile immuable $»^{21}$, mobile car susceptible de circuler d'un endroit à l'autre, immuable car produisant de façon sûre un résultat identique. Dans le cas des technologies de démocratie, la standardisation repose sur des textes de droit, des 
habitudes de pratiques, des formats d'instruction publique, des instruments de circulation de l'information, etc. Elle permet aujourd'hui à la technologie électorale, après les incertitudes des expérimentations initiales ${ }^{22}$, de réaliser ses objectifs - fournir un vainqueur de l'élection sans subir des contestations à chaque réplication. Dans le cas des technologies de démocratie, la standardisation va avec l'institutionnalisation, comprise comme l'intégration dans les institutions démocratiques telles qu'elles sont stabilisées par des textes de droit.

Les exemples cités plus haut fournissent d'autres illustrations de standardisation (encore en cours). La Commission Nationale du Débat Public, grâce à son inscription dans le droit, à la formalisation des méthodes qu'elle adopte pour la mise en débat des projets d'infrastructure, à la réflexivité permanente des responsables de la CNDP sur leurs pratiques, et à l'intégration de l'exigence du débat public dans la conduite des projets des aménageurs est aujourd'hui en train de connaître une telle standardisation ${ }^{23}$.

Le travail des chercheurs visant à donner sens à l'expérience du « débat public à la française », voire à fournir des critères d'évaluation de celle$\mathrm{ci}^{24}$, peut contribuer à ce processus. Malgré des propositions de formalisation juridique ${ }^{25}$, un appui sur un corpus de textes de sciences sociales utilisés comme références et un usage de plus en plus répandu, la conférence de citoyens n'en est pas à ce stade. Sa circulation dessine cependant des espaces relativement homogènes, notamment par l'intermédiaire d'acteurs privés comme l'IFOP, dans lesquels la parole du « citoyen neutre » prend de plus en plus d'importance.
La standardisation permet la circulation des instruments et le succès des "épreuves de réplication ", c'est-à-dire des tentatives de reproduction d'une technologie de démocratie sur un nouveau sujet. Les investissements nécessaires au passage de l'épreuve de réplication sont visibles lorsqu'on considère des cas difficiles. Une illustration évocatrice est l'expérience de pensée de Peter Sloterdijk consistant à parachuter un « parlement gonflable » en Irak, en espérant réaliser la démocratie libérale du simple fait de la réplication d'une technologie de démocratie certes cruciale, mais coupée des réseaux de standards et de pratiques nécessaires à son fonctionnement ${ }^{26}$. Le cas des nanotechnologies fournit d'autres exemples parlant d'épreuves de réplication des expériences démocratiques. Elles concernent à la fois les protagonistes des expériences comprises comme assemblages incertains et les experts des technologies de démocratie. Les premiers, expérimentant des modes de décisions collectives et de traitement des problèmes des nanotechnologies, peuvent ensuite tenter d'étendre l'expérience acquise. On peut comprendre ainsi les tentatives des initiateurs de la norme «nano-responsable » de poursuivre le projet dans les instances européennes, voire internationales, de la standardisation. Dans ce cas, l'expérience initiale est conçue comme une première étape avant la fabrication d'un instrument susceptible de circuler parmi l'ensemble des acteurs concernés par la production des nanomatériaux. L'extension de l'expérience comme assemblage incertain peut aussi consister à tenter la réplication, et donc à faire de l'instrument une technologie de démocratie séparable des questions sur lesquelles elle est appliquée. Ainsi, de récents travaux 
destinés aux pouvoirs publics recommandent l'application du modèle du Nanoforum à la biologie de synthèse ${ }^{27}$. Ces opérations peuvent être comprises comme des situations de bifurcation entre l'expertise politique (celle des experts des technologies de démocratie) et d'autres formes d'expertise (notamment, sur des questions comme les nanotechnologies ou la biologie de synthèse, l'expertise scientifique) ${ }^{28}$. Pour les experts des technologies de démocratie, réussir les épreuves de réplication est une condition nécessaire pour assurer la validité de l'expérience. Ceci n'empêche pas que certains d'entre eux identifient une nécessité d'innovation dans les procédures utilisées, en utilisant par exemple les outils de délibération en ligne, qui redéfinissent les modes d'intervention des publics $^{29}$. Mais les experts des technologies de démocratie aux prises avec la réplication de leurs dispositifs doivent aussi être capables de résoudre ce qu'Harry Collins appelle la « régression de l'expérimentateur », c'est-à-dire l'impossibilité de différencier l'erreur de mesure de l'échec de l'expérience sans faire référence à une connaissance dont on sait à l'avance qu'elle est valide $^{30}$. Par exemple, dans le cas de la conférence de citoyens, les organisateurs doivent pouvoir différencier la parole du citoyen «neutre » de celle du citoyen « non neutre », afin de pouvoir repérer « l'erreur expérimentale » que serait la sélection d'un « mauvais citoyen ». Effectuer cette différenciation suppose de savoir à l'avance ce que sera la parole du citoyen neutre, et donc le résultat de l'expérience. Ainsi, les experts de la conférence de citoyens sont capables de repérer les situations où un citoyen se comporte mal, et doit donc être considéré comme une « erreur de recrutement ». Le cas s'est présenté lors de la conférence sur les nanotechnologies commanditée par le conseil régional de l'îlede-France : les organisateurs ont réalisé après coup avoir commis « une erreur de casting », en incluant dans le panel (malgré un processus de sélection sophistiqué) un citoyen refusant en bloc les nanotechnologies, et critiquant le principe même de la consultation des publics. Dans ce cas, les frontières de la neutralité définie par les experts de la conférence étaient franchies : la parole de ce citoyen ne pouvait être celle du «public neutre », mais n'était qu'une erreur expérimentale. Il a alors fallu faire avec cette difficulté additionnelle pour parvenir malgré tout à produire des recommandations raisonnables. $\mathrm{Ce}$ cas est, à bien des égards, exceptionnel, car il met en évidence une situation où un membre du panel approche une situation de rupture. La situation est différente dans nombre de jurys citoyens, à propos desquels des travaux ont montré la complexité du rôle du «bon citoyen » et les micro-arrangements impliquant organisateurs et membres des panels pour permettre l'émergence de la délibération en petit groupe ${ }^{31}$. Mais l'explicitation de l'affrontement dans le cas décrit ici (liée pour une part à la diversité des problématisations possible des nanotechnologies) permet de mettre au jour l'enjeu crucial qu'est l'épreuve de réplication.

Dans le cas du débat public CNDP, les organisateurs sont censés savoir si des participants à un débat public interviennent de façon « non acceptable ", et peuvent adapter le dispositif pour tenter d'éliminer ceux qui refusent les règles de la discussion. Là encore, la réplication de l'instrument sur les nanotechnologies a donné lieu à une "erreur expérimentale », 
liée à l'intervention de publics critiques des nanotechnologies et de leur mise en discussion publique. Les réunions publiques du débat CNDP ont fourni à des opposants aux nanotechnologies une opportunité d'action. Pour ces opposants, qui contestent un programme de développement technologique compris comme visant au contrôle de l'homme et de la nature, et comprenant l'enrôlement du public pour assurer sa réalisation, le débat public n'était qu'une manœuvre destinée à faire accepter un développement technologique non discutable, au prix, dans le meilleur des cas, de la gestion de ses conséquences néfastes (notamment en termes de risques sanitaires). Du point de vue des organisateurs, le dispositif se trouve face à une difficulté : comment faire avec un public qui est concerné par le sujet, et doit donc être impliqué dans le débat, mais dont le point de vue refuse la validité de la technologie de démocratie elle-même. Trancher ce problème n'est pas si évident, alors que chacun des débats CNDP donne lieu à un « débat sur le débat », selon l'expression consacrée ${ }^{32}$, qui consiste à mettre en discussion les objectifs, les modalités et l'opportunité même du dispositif. Les opposants aux nanotechnologies ont été jugés au final comme des publics dont l'intervention ne pouvait être incluse (et ne souhaitait pas l'être) dans le dispositif. Celui-ci devait donc être adapté afin d'exclure des interventions dont les organisateurs prévoyaient qu'elles auraient perturbé le déroulement de l'expérience, et donc créé un artefact cachant la réalité des opinions des publics légitimes. Ainsi, la commission organisatrice du débat a tenté de séparer les réunions en deux salles, l'une fermée, dans laquelle se trouvaient les acteurs invités et les organisateurs, et l'autre publique, qui pouvait contenir les interventions des opposants. Les deux dernières réunions du débat CNDP ont ensuite été tenues dans un lieu tenu secret, et révélé seulement le jour même de la réunion aux participants préalablement inscrits.

Le cas des nanotechnologies est d'autant plus intéressant pour analyser les épreuves de réplication que les incertitudes sur les objets eux-mêmes et sur les programmes rendent les expériences fondées sur les technologies de démocratie complexes à réaliser. Ce cas impose des épreuves relatives aux objets et aux publics, et, par là même, conduit à expliciter les questions que posent les modalités de l'expérience démocratique. Les nanotechnologies sont en effet un programme de politique scientifique associant, par l'intermédiaire des plans de financement et des feuilles de route, les acteurs publics et privés dans la réalisation d'objectifs qui tiennent à la fois à la redéfinition des pratiques scientifiques et industrielles existantes et à l'espérance d'innovations radicales à plus ou moins long terme. À ce titre, la définition des nanotechnologies est elle-même un enjeu, puisqu'il s'agit de définir des objectifs de développement, et l'extension de ce qui recevra un soutien public ou un financement provenant d'investissements privés. À cela s'ajoute l'incertitude sur les « objets nano » eux-mêmes. La définition des «nanomatériaux » fait l'objet de nombreuses discussions au sein des instances de régulation nationales et européennes et dans les institutions de standardisation internationales. Et choisir un critère plutôt qu'un autre pour définir les nanomatériaux (par exemple, un critère de taille plus ou moins étendue) a des conséquences énormes, car cela revient à inclure ou exclure 
un ensemble de substances du périmètre d'une réglementation future.

En conséquence, l'application aux nanotechnologies de technologies de démocratie fondées sur la représentation objective de la science pour un public de citoyens neutres se heurte à de nombreuses difficultés. Au cours de la conférence de citoyens sur les nanotechnologies, les organisateurs de l'IFOP ont dû faire face aux réticences de certains membres du comité de pilotage, qui n'acceptaient pas la représentation « objective » des nanotechnologies sous la forme d'une liste d'applications dont des experts pourraient déterminer les bénéfices et les risques. En insistant sur la définition des nanotechnologies comme programme en développement, ces membres proposaient d'amender l'instrument pourtant maitrisé par les experts de l'IFOP, en refusant de considérer la formation des membres du panel aux questions discutées comme un problème de vulgarisation scientifique.

La réplication du débat public de la CNDP sur les nanotechnologies a fait elle-même face aux particularités de l'entité nanotechnologies. La Commission est devenue experte dans l'organisation de débats sur des projets d'infrastructure, au cours desquels elle peut apparaître comme une instance organisant l'explicitation des arguments et, par là, l'exploration de la controverse. Mais la CNDP a dû adapter son dispositif pour assurer son fonctionnement sur les nanotechnologies ${ }^{33}$. Comme le domaine couvre potentiellement tout le territoire pour une large étendue d'applications industrielles, la Commission particulière chargée de l'organisation du débat a choisi de tenir des réunions publiques dans toute la France, de définir un thème principal pour chacune d'elles en fonction des activités industrielles locales - par exemple «nanotechnologies et cosmétiques » lors d'une réunion à Orléans, où se trouve une usine l'Oréal - tout en laissant la possibilité aux participants d'aborder n'importe quel sujet lié aux nanotechnologies. Cette adaptation du dispositif CNDP (au départ conçu pour des discussions sur des projets d'infrastructure locale) n'empêchait pas les organisateurs de se heurter au problème du repérage des objets et des acteurs des nanotechnologies. Dans la mesure où la définition des « nano » n'est pas tranchée, des industriels pouvaient affirmer qu'ils « ne faisaient pas de nano ", sans que la CNDP ne parvienne à mettre en discussion ce qui apparaissait alors comme un enjeu politique central, c'est-à-dire la définition de ce qui peut être caractérisé comme «nano».

En analysant les tentatives de réplication des technologies, on peut ainsi repérer quels sont les « scripts $»^{34}$ des technologies de démocratie, c'est-à-dire quel type d'usages elles requièrent, et quelles situations leur permet de fonctionner au mieux. Les épreuves de réplication permettent de repérer ce que ne parviennent pas à saisir les technologies de démocratie censées circuler d'un domaine à l'autre. Ainsi, les problèmes de la définition des programmes et des objets des nanotechnologies sont discutés dans des dispositifs comme le Nanoforum ou la norme nano-responsable, qui ne sont pas pensés comme des instruments dont la validité est indépendante du cas particulier " nanotechnologies ». Mais les épreuves de réplication sont aussi les étapes nécessaires à la solidification de l'instrument et de ses usages. Dans les cas traités ici, elles 
ont été franchies avec un succès relatif. Les commanditaires de la conférence de citoyens sur les nanotechnologies ont jugé que les objectifs avaient été atteints : des recommandations raisonnables ont été produites par un panel de citoyens. Les commanditaires du débat public CNDP sont plus ambivalents : la position du gouvernement suite au rapport du débat a été rendue publique en février 2012, plus d'un an après la fin du processus. Elle annonce qu'à la suite du débat, le gouvernement cherchera à « mieux apprécier et prévenir les risques», « informer le public » et à « associer la société civile à la gouvernance des nanotechnologies». Quant à l'influence à long terme sur la capacité de la CNDP à fournir au gouvernement un outil fiable pour l'organisation de débats sur des options générales de politique publique, elle ne peut être tranchée à l'heure actuelle.

\section{Conclusion}

À partir d'une interrogation sur la description des initiatives participatives en termes d' " expériences », cet article a proposé une analyse des pratiques démocratiques prenant pour objet les dispositifs qui les réalisent. Des exemples liés aux nanotechnologies ont permis de décrire deux modalités de l'expérience démocratique. D'une part, l'expérience comprise, sur le modèle de la philosophie pragmatiste, comme la situation dans laquelle le monde s'invente au sein d'un assemblage à l'issue incertaine. D'autre part, l'expérience comme mobilisation d'un instrument participatif qui peut être considéré comme une " technologie de démocratie », sur le modèle des technologies scientifiques. Les deux modalités de l'expérience réalisent deux formes politiques distinctes. La première est marquée par la découverte progressive des contraintes, et l'incertitude sur la transformation possible des processus de prises de décision collective. La seconde est caractérisée par une organisation sociale laissant à des experts le soin de produire les publics dont les avis doivent être mesurés et dont la légitimité est définie par les caractéristiques de l'instrument utilisé (par exemple la «neutralité » dans le cas de la conférence de citoyens).

Les exemples décrits ici sont relatifs aux nanotechnologies, un domaine très spécifique, qui a conduit à des initiatives originales (comme le Nanoforum ou la norme « nano-responsable) et à introduire des modifications notables dans des dispositifs existants (comme le débat CNDP). Mais du fait même des spécificités des nanotechnologies, cet article a pu mettre en lumière deux modalités très différenciées de l'expérience démocratique, et rendre visible, sur des cas extrêmes, l'importance des épreuves de réplication pour la circulation des technologies de démocratie. La description des deux modalités de l'expérience souligne l'intérêt analytique des opérations permettant d'isoler, à partir d'une expérience comme assemblage incertain local, une technologie de démocratie ensuite susceptible de réplication. Franchir des épreuves de réplication est une condition pour permettre à la technologie de démocratie de circuler. Les difficultés rencontrées lors de la réplication depuis d'autres questions vers les nanotechnologies rendent visibles les investissements nécessaires à l'expérience fondée sur des technologies de démocratie comme la conférence de citoyens ou le débat CNDP, et les alternatives qu'elle 
élimine. Par contraste, l'expérimentation comme assemblage permet aux acteurs impliqués de saisir la nature fondamentalement incertaine des objets et des programmes des nanotechnologies. Mais les exemples examinés ici sont aussi des cas où les contraintes multiples relatives aux caractéristiques des objets discutés et aux intérêts des acteurs impliqués ne permettent pas de transformer l'assemblage expérimenté en mécanisme contraignant (en mettant en place, par exemple, une certification).

Ce type d'analyse fournit un répertoire de description utile pour rendre compte des situations où l'interrogation sur la démocratie est inséparable de la prise de décision collective et donne lieu à la mobilisation de technologies de démocratie. Il conduit à poursuivre la critique de la « démocratie telle qu'elle existe réellement $»^{35}$ en explicitant, d'une part, les formations politiques produites par les instruments participatifs qui circulent sous la forme de technologies de démocratie, et, d'autre part, en explicitant des choix alternatifs : ceux que proposent les critiques des technologies de démocratie expérimentées comme ceux qui ne circulent pas en dehors d'une expérience locale non répliquée ni standardisée.

Associer l'analyse des circulations des technologies de démocratie et les expériences d'assemblages locaux permet de rendre compte de la constitution d'espaces relativement homogènes, caractérisés par des modalités de conduite d'expériences et de démonstration. Ceux-ci sont directement liés aux formes stabilisées de production de la légitimité politique et de l'objectivité scientifique (ce que Sheila Jasanoff décrit par la notion d'« épistémologie civique $\left.»^{36}\right)$. Les trajectoires des technologies de démocratie rencontrent, à cet égard, des frontières géographiques, qu'elles peuvent contribuer à solidifier. Ainsi, l'usage de la conférence de citoyens sur les nanotechnologies, en France, est fondée sur la démonstration des capacités du citoyen neutre à contribuer à la discussion publique de façon rationnelle. Aux ÉtatsUnis, elle est un outil utilisé, dans le cas des nanotechnologies, par l'expertise en sciences sociales pour connaître les mécanismes de délibération en petits groupes ${ }^{37}$. La comparaison entre les espaces politiques que dessinent les trajectoires des technologies de démocratie est alors un résultat de l'analyse, qui doit s'appuyer sur la généalogie des expériences précédentes et sur l'étude des modalités de l'expertise sur la démocratie, qui peut être le fait d'acteurs publics ou privés.

Ce type d'étude permet d'analyser la légitimité démocratique comme un résultat du travail des acteurs, qui dépend de leur capacité à réussir la construction des publics par les dispositifs qu'ils mettent en place. Les contre-expériences que proposent les acteurs critiques montrent que l'ordre politique que tentent de mettre en forme les technologies de démocratie n'est pas le seul possible, et que donc la légitimité démocratique n'est pas acquise. La démocratie apparaît alors comme l'objet même de l'analyse, dont on peut rendre compte par l'analyse des dispositifs qui la construisent et des épreuves successives que ces dispositifs rencontrent. Ce travail d'explicitation met au jour la variété des formes de l'ordre démocratique. Il ne mobilise pas de critère d'évaluation pour qualifier le caractère plus ou moins démocratique mais met 
au jour la répartition des rôles entre « experts » et « profanes » des technologies de démocratie, et l'organisation politique que réalise leur circulation. L'analyse est critique, au sens où elle rend visibles les choix politiques, relatifs à la définition des publics et des problèmes à traiter, contenus dans les instruments, et les investissements nécessaires à leur stabilisation. On peut alors considérer comme une entreprise de « démocratisation » l'augmentation des possibilités de désaccords sur les expériences démocratiques, y compris donc sur les ressorts de la construction des publics, des problèmes et de la légitimité de la décision collective.

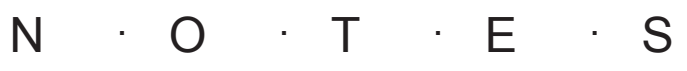

1. M. Revel et al., Le débat public, une expérience française de démocratie participative, Paris, La Découverte, 2007.

2. L'expression était utilisée lors d'une conférence en juin 2007 par Marc Lipinski, élu au conseil régional de l'île-de-France, alors qu'il commentait les résultats d'une conférence de citoyens sur les nanotechnologies dont il avait eu l'initiative.

3. N. Rose, Powers of Freedom, Cambridge, Cambridge University Press, 1999.

4. A. Garrigou, "Le secret de l'isoloir», in Actes de la Recherche en Sciences Sociales, vol. 71, n71-72, 1998, pp. 22-45.

5. M. Lynch, « Pandora's Ballot Box: Comments on the 2000 US Presidential Election », Social Studies of Science, vol. 31, n³, 2001, pp. 417-419.; C. Miller, 2006, «Interrogating the civic epistemology of the American democracy. Stability and instability in the 2000 U.S. presidential election », Social Studies of Science, vol. 34, n4, 2006, pp. 501-530.

6. Je ne reprends donc pas à mon compte la catégorie de la « démocratie expérimentale » utilisée pour caractériser (et parfois défendre) un régime définissant des objectifs généraux pour laisser à la négociation locale le soin de leur mise en œuvre. Cf. C. Sabel, « Révolution tranquille de la gouvernance démocratique : vers une démocratie expérimentale » in W. Michalski et al. (dir.), La gouvernance au XXI siècle, Paris, OCDE, 2002, pp. 141-176.

7. Cet article utilise des matériaux empiriques discutés dans un ouvrage et une thèse. Cf. B. Laurent, Les politiques des nanotechnologies. Pour un traitement démocratique d'une science émergente, Paris, Charles Léopold Mayer, 2010 ; B. Laurent, Brice, «Technologies of Democracy. Experiments and 
Demonstrations », Science and Engineering Ethics, vol. 17, n³, 2011, pp. 649-666.

8. Le dispositif de la « conférence de citoyens », centré sur le travail d'un panel de citoyens sélectionnés pour produire un avis sur une question technique donné à la suite d'une formation qui leur est délivrée, est aujourd'hui bien connu. Pour une description largement mobilisée par les promoteurs du dispositif Cf. D. Bourg, D. Boy, Conférences de citoyens. Mode d'emploi, Paris, Descartes, 2000.

9. Pour une mise en perspective de ce débat au sein de la trajectoire d'institutionnalisation du débat public «à la française », Cf. J-M. Fourniau «L'institutionnalisation controversée d'un modèle français de débat public », in Téléscope, vol. 17, n 1, 2011, pp. 70-93.).

10. C. Lefort, Essais sur le Politique, Paris, Seuil, 1986, p. 25-30. On peut rapprocher cette perspective de celle de Pierre Rosanvallon pour qui la démocratie « consiste d'abord dans une expression saine et ouverte des conflits d'intérêts et des différends d'appréciation », in P. Rosanvallon, Pierre, La légitimité démocratique. Impartialité, réflexivité, proximité, Paris, Seuil, 2008, p. 190

11. Grenoble est un site important de la recherche française et européenne en nanotechnologies. C'est là également que la contestation des nanotechnologies a été la plus vive.

12. Ce sont les termes de l'initiatrice du dispositif, employée de la DGS (extrait de carnet de terrain).

13. Ce projet est devenu un des éléments de la position française lors de sa candidature (réussie) à la présidence et au secrétariat du comité " nanotechnologies » du CEN.

14. S. Shapin, S. Schaffer, Leviathan and the air-pump, Princeton, Princeton University Press, 1985

15. La constitution d'objet grâce au dispositif expérimental (le vide dans la pompe à air) est d'ailleurs un des fondements de la critique de l'adversaire de
Boyle, Hobbes, pour qui des réalisations artéfactuelles ne peuvent prétendre révéler quoi que ce soit de la nature.

16. C'est la perspective adoptée par Javier Lezaun et Linda Soneryd dans un article où ils décrivent des « technologies d'élicitation » (in J. Lezaun, Javier, L. Soneryd, 2007, « Consulting citizens: technologies of elicitation and the mobility of public », Public Understanding of Science, vol. 16, n³, 2007, pp. 279297) permettant de « rendre les communautés réelles » (N. Rose, op. cit., pp. 189-190).

17. J'utilise le terme «technologie de démocratie » plutôt que « technologies de participation », dans la mesure où je ne cherche pas à isoler un domaine qui serait celui de la "participation » (par rapport, par exemple, aux activités qui seraient celle de la démocratie représentative). Les technologies de démocratie peuvent désigner aussi bien des procédures dites «participatives » que celle de la démocratie représentative.

18. Le film est aujourd'hui utilisé comme support d'enseignement pour montrer à des étudiants l'intérêt du dispositif de la conférence de citoyens. Il faisait partie d'un important plan de médiatisation, et signale des activités de démonstration également dirigées vers l'interne du conseil régional ; Cf. G. Gourgues, Le consensus participatif. Les politiques de la démocratie dans quatre régions françaises, thèse de doctorat, Université de Grenoble, 2010, pp. 1042-1044.

19. En entretien, un employé se félicite ainsi que le laboratoire pharmaceutique pour qui l'IFOP organise régulièrement des conférences de citoyen soit maintenant convaincu que le « dispositif fournisse des avis mesurés », après une crainte initiale d'obtenir des résultats « inapplicables car trop radicaux» (par exemple sur la refonte totale du système de santé publique) (entretien, Paris, février 2009).

20. Notamment parce qu'elles concernaient des 
questions traitées à d'autres niveaux administratifs et politiques.

21. B. Latour, 1989, La Science en Action, Paris, La Découverte, 1989 ; B. Latour, « Drawing things together », in M. Lynch, Michael, S. Woolgar (eds.), Representations in scientific practice, Cambridge, MIT Press, 1990, p. 19-68 ; A. Mallard, , 1998, « Compare, Standardize and Settle Agreement. On some usual metrological problems », Social Studies of Science, vol. 28, n4, 1998, pp. 571-601.

22. A. Garrigou, op. cit., 1998

23. Pour un panorama du dispositif et de ces modes d'action Cf. M. Revel, op. cit.

24. M. Brugidou et al., 2007, "Quels critères d'évaluation du débat public? Quelques propositions », in Revel et al., op. cit., 200, pp. 305-317.

25. F. Jacquemot, Des conférences de citoyens en droit français, Rapport de projet PICRI, Conseil Régional d'Île-de-France, 2007.

26. P. Slotedijk, G. von der Hagen, « Instant democracy: the pneumatic parliament », in B. Latour, P. Weibel (dir.), Making things public, Cambridge, MIT Presse, 2004, pp. 952-959.

27. P-B. Joly, et al., Biologie de synthèse. Conditions d'un dialogue avec la société, Paris, Rapport pour le ministère de 1'Enseignement supérieur et de la Recherche, 2011.

28. On pourrait ainsi décrire ces cas de bifurcation dans les termes qu'emploie Bruno Latour lorsqu'il analyse les processus de purification de la science et de la politique, notamment à propos de l'exemple de Boyle Cf. B. Latour, 1993, Nous n'avons jamais été modernes, Paris, La Découverte.

29. N. Benvegnu, «Le débat public en ligne. Comment s'équipe la démocratie dialogique », in Politix, vol. 3, n 75,2006 , pp. 103-124.

30. H. Collins, Changing orders, Londres, Sage, 1985. 31. J. Talpin, « Jouer les bons citoyens. Les effets contrastés de l'engagement au sein des dispositifs délibératifs », in Politix, vol. 3, n 75 , 2006, pp. 11-31. 32. J-M. Fourniau, «L'expérience démocratique des "citoyens en tant que riverains" dans les conflits d'aménagement », Revue européenne des sciences sociales, XLV, n¹36, 2007, pp. 149-179.

33. Le débat CNDP sur les nanotechnologies est un débat sur des « options générales ». Permise depuis la loi « démocratie de proximité » de 2002, cette version du débat CNDP étendue aux options de politique publique est encore rare.

34. M. Akrich, «The description of technical objects », in W. Bijker, J. Law (Eds.), Shaping Technology/ Building Society: Studies in Sociotechnical Change, Cambridge, MA, MIT Press, 1992, pp. 205-224.

35. N. Fraser, «Rethinking the Public Sphere: A Contribution to the Critique of Actually Existing Democracy », Social Text, n²5/26, 1990, pp. 56-80.

36. S. Jasanoff, Designs on Nature. Science and Democracy in Europe and the United States, Princeton, Princeton University Press, 2005.

37. B. Laurent « Replicating participatory devices. The consensus conference confronts nanotechnology ", CSI working paper $n^{\circ} 11,2009$. Ce dernier exemple invite à contraster l'approche proposée ici pour l'étude des expériences démocratiques avec les méthodes de sciences sociales fondées sur l'expérimentation, sur le modèle des sciences naturelles, et qui sont largement employées dans le cas de l'étude de la délibération, et ce dès les années 1970 Cf. J. Lezaun, « Offshore democracy : launch and landfall of a sociotechnical experiment ", in Economy and Society, vol. 40, $\mathrm{n}^{\circ} 4$, 2011, pp. 553-581. L’approche revendiquée ici s'attache à décrire les expériences démocratiques plutôt qu'à en réaliser en milieu contrôlé, sur le modèle de l'expérience scientifique. Elle ne s'interdit pourtant pas de mettre à l'épreuve la distance du chercheur à son objet, en fonction des modalités de l'expérience 
étudiée. En ce sens, elle est «pragmatiste», au sens des expérimentateurs pragmatistes de la première modalité de l'expérience décrite ici. Ce texte n'a pas cherché à discuter les modalités de l'intervention du chercheur étudiant les expériences démocratiques, ce qui mériterait un développement spécifique.

\section{$\mathrm{R} \cdot \dot{E} \cdot \mathrm{S} \cdot \mathrm{U} \cdot \mathrm{M} \cdot \dot{E}$}

Cet article se penche sur la notion d'" expérience » en matière de démocratie participative. L'analyse est fondée sur le cas des nanotechnologies, où l'incertitude sur les objets et sur les publics permet de mettre au jour deux modalités de l'expérience démocratique. D'une part, l'expérience comprise comme un assemblage dans laquelle la réalité technique et sociale s'invente. D'autre part, l'expérience comme mobilisation d'une « technologie de démocratie », dont la mise en œuvre fait appel à des experts. Le passage d'une modalité à l'autre exige de réussir des épreuves de réplication, au cours desquelles les technologies de démocratie peuvent être distinguées des particularités des situations sur lesquelles elles sont appliquées. Cette approche permet de mettre au jour les opérations de construction de la légitimité démocratique, en ouvrant à l'examen critique la politique des expériences démocratiques.

\section{Abstract}

This paper focuses on the notion of " experience » in participatory democracy. The analyis is based on the case of nanotechnology, a domain where the uncertainty about both the objects and publics makes visible two modalities of the democratic experience. On the one hand, the experience as an assemblage where technical and social realities are uncertain. On the other hand, the experience connected to expertbased «technologies of democracy ». Going from one modality to the other requires to pass replication trials, within which technologies of democracy are separated from the particularities of the situations where they are applied. This approach displays the operations that produce democratic legitimacy, while also opening to critical analysis the politics of democratic experiences. 